\title{
DAS DEUTSCHE INSTITUT FÜR AFRIKA-FORSCHUNG
}

Die Afrika-Forschung ist in Deutschland lange Zeit vernachlässigt worden. Rein fachwissenschaftliches Vorgehen hatte den Vorrang. Eine Ausnahme bildeten die wenigen Disziplinen, die durch ihre Aufgabenstellung auf Afrika bezogen sind. In den übrigen Disziplinen wurden afrikanische Fragestellungen im allgemeinen nur am Rande fachlicher Untersuchungen behandelt. Diese Konzeption reichte nicht aus, um die Entwicklung des afrikanischen Kontinents in ihrer Eigenständigkeit erfassen zu können. Besonders deutlich zeigte sich ihre Schwäche bei der Behandlung gegenwartsbezogener und praxisnaher Fragen über Afrika, die ohne eine vertiefte Regionalkenntnis nicht befriedigend beantwortet werden können. Deshalb hat das Deutsche Institut für Afrika-Forschung (DIAF) in Hamburg Anfang 1963 seine Arbeit mit dem Ziel begonnen, durch systematische Bearbeitung dieser regionalen Aspekte die deutsche Afrika-Forschung zu fördern. Anregungen gaben hierfür interdisziplinäre afrikawissenschaftliche Studien, die vor allem in den USA und in der Sowjetunion, in Belgien, Frankreich und Großbritannien sowie in verschiedenen afrikanischen Ländern zu neuen Erkenntnissen führten.

Das DIAF steht mit dem Afrika-Verein e. V., Hamburg, in enger Verbindung. Im Rahmen des Deutschen Übersee-Institutes, Hamburg, arbeitet es zugleich mit den drei anderen, in Hamburg bestehenden Regional-Instituten ${ }^{1}$ zusammen. Das DIAF ist als eingetragener Verein konstituiert. Die Organe des Vereins sind der Aufsichtsrat, der Vorstand und die Mitgliederversammlung. Der Aufsichtsrat besteht aus Persönlichkeiten der deutschen Afrika-Wirtschaft und gewährleistet dadurch einen engen Kontakt zur Praxis. Der Vorstand wird vom Aufsichtsrat bestellt und ist für die laufende Geschäftsführung verantwortlich, die in Personalunion mit dem Afrika-Verein wahrgenommen wird. Die höchstens 25 ordentlichen Mitglieder aus Wissenschaft und Praxis umfassende Mitgliederversammlung bestimmt auf Vorschlag des Vorstandes und der Geschäftsführung die Ausrichtung des Forschungsprogrammes.

Aufgabe des DIAF ist die interdisziplinäre, praxisnahe Afrika-Forschung. Den Vorrang haben die Sozialwissenschaften. Neben den ökonomischen sollen insbesondere auch rechtliche, soziologische und zeitgeschichtliche Probleme untersucht und beobachtet werden.

Hierfür steht dem Institut ein kleiner Stab festangestellter Fachwissenschaftler zur Verfügung, der aus zwei Politologen sowie einem Geographen, einem Historiker, einem Juristen und einem Volkswirt besteht. Er wird durch eine wechselnde Zahl freier Mitarbeiter aus verschiedenen Disziplinen (z. Z. ein Afrikanist, drei Geographen, ein Zeitungswissenschaftler, zwei Zoologen) ergänzt, die mit bestimmten Forschungsaufträgen betraut sind.

Die Ergebnisse der Forschungsvorhaben werden in der Reihe HAMBURGER BEITRAGE ZUR AFRIKA-KUNDE veröffentlicht. Seit 1966 wird außerdem dreimal jährlich die Zeitschrift AFRIKA SPECTRUM herausgegeben. Jedes Heft ist einem besonderen Thema gewidmet und enthält die laufende Dokumentation "Aus Afrikanischen Gesetzesblättern" sowie Informationen und Buchbesprechungen. Das Institut ist Mitherausgeber der Zeitschrift VERFASSUNG UND RECHT IN UBERSEE sowie Mitarbeiter am AFRIKA-HANDBUCH und am Mitglieder-

1 Deutsches Orient-Institut, Institut für Asienkunde, Institut für Iberoamerika-Kunde. 
Rundschreiben des Afrika-Vereins, das unter dem Titel AFRIKA - INFORMATIONEN AUS WIRTSCHAFT, POLITIK UND RECHT erscheint.

Um den Erfahrungsaustausch zwischen Wissenschaftlern und Praktikern zu fördern und das interdisziplinäre Gespräch $\mathrm{zu}$ pflegen, wird in regelmäßiger Folge in Hamburg das COLLOQUIUM veranstaltet.

Die BIBLIOTHEK umfaßte Anfang 1968 über 6500 Einheiten Afrika-Literatur mit den Schwerpunkten Wirtschaft und Politik. Es werden etwa 275 Zeitungen, Zeitschriften und andere Periodika, darunter 45 Amtsblätter der afrikanischen Staaten, gesammelt. In seiner Arbeit kann sich das DIAF zusätzlich auf die Erfahrungen und die umfassenden Afrika-Bestände Hamburger Institute und Bibliotheken stützen², die sich Ende 1966 zur ARBEITSGEMEINSCHAFT DER HAMBURGER, AN AFRIKA INTERESSIERTEN FORSCHUNGSINSTITUTE zusammenschlossen. Sie verfügen über ein in dieser Breite an keiner anderen Stelle in der Bundesrepublik vorhandenes Potential an Afrika-Kenntnissen und -Material. Deswegen wurde das DIAF 1967 mit dem Aufbau der deutschen DOKUMENTATIONSLEITSTELLE FÚR AFRIKA betraut, die in enger Zusammenarbeit mit dem Institut für Dokumentationswesen in Frankfurt, dem Bundesministerium für Wirtschaftliche Zusammenarbeit und der Deutschen Stiftung für Entwicklungsländer sowie mit den drei anderen Hamburger Regional-Instituten erfolgt, die ähnliche Zentralen für ihre jeweilige Region errichten.

Aufgabe dieser Dokumentationsstelle ist es, einschlägige Informationen über alle Afrika (außer Ägypten ${ }^{3}$ ) betreffende Sachgebiete auszuwerten, zu speichern und zu vermitteln. Hierbei bedient sie sich verschiedener Arbeitsmittel. Am wichtigsten ist noch immer die konventionelle bibliographische Steilkartei, deren Grundbestand der 11700 Karten umfassende Karteidienst des Centre International de Documentation Economique et Sociale Africaine (CIDESA) in Brüssel und die Karten mit den Afrika-Neuzugängen des Hamburgischen Welt-Wirtschafts-Archivs (Bestand etwa 1300), des Instituts für Weltwirtschaft in Kiel (Bestand etwa 50 000) und der Staats- und Universitätsbibliothek Hamburg bilden. Außerdem erhält sie die Karteidienste des Centre d'Analyse et de Recherches Documentaires pour l'Afrique Noire (CARDAN) in Paris und des Secrétariat d'Etat aux Affaires Etrangères Chargé de la Coopération in Paris. Die in eigener Produktion hergestellten Karteikarten, die neben Monographien, Teilen aus Sammelwerken und Reportliteratur hauptsächlich Zeitschriftenaufsätze verzeichnen, werden unter verschiedenen Gesichtspunkten abgestellt. Ab Mitte 1968 sollen sie auf Lochstreifen aufgenommen werden, um die spätere Datenverarbeitung vorzubereiten. Außer der bibliographischen Steilkartei befinden sich verschiedene andere, darunter insbesondere die Institutionen- und die Projektkartei im Aufbau. Sie werden durch einen umfangreichen bibliographischen Handapparat ergänzt.

Die Aufbauphase des DIAF konnte dank der Zuwendungen aus den Mitteln der Deutschen Übersee-Stiftung, Hamburg, 1965 im wesentlichen abgeschlossen werden.

\footnotetext{
2 Der Gesamtbestand der Afrika-Literatur in Hamburg umfaßte nach einer Mitte 1966 durchgeführten Umfrage rund 100000 Bände bzw. akzessorische Einheiten. Er verteilt sich auf die folgenden Institute und Bibliotheken in Hamburg: Bernhard-Nocht-Institut für Schiffs- und Tropenkrankheiten, Bundesforschungsanstalt für Fischerei, Bundesforschungsanstalt für Forst- und Holzwirtschaft, Commerzbibliothek, Deutsches Hydrographisches Institut, Forschungsstelle für Völkerrecht und ausländisches öffentliches Recht und Institut für Auswärtige Politik, Geologisches Staatsinstitut, Hamburgisches Museum für Völkerkunde und Vorgeschichte, Hamburgisches Welt-Wirtschafts-Archiv, Historisches Seminar der Universität Hamburg, Institut für Außenhandel und Uberseewirtschaft Hamburg, Institut für Geographie und Wirtschaftsgeographie, Max-Planck-Institut für ausländisches und internationales Privatrecht, Missionswissenschaftliches Seminar der Universität Hamburg, Seewetteramt des Deutschen Wetterdienstes, Seminar für afrikanische Sprachen und Kulturen, Staats- und Universitätsbibliothek Hamburg, Staatsinstitut für Allgemeine Botanik, Staatsinstitut für Angewandte Botanik, Unesco-Institut für Pädagogik, Zoologisches Staatsinstitut.

3 Nach einer Vereinbarung der interessierten Hamburger Institutionen wird Ägypten vom Deutschen OrientInstitut und vom Nah- und Mittelost-Verein bearbeitet.
} 
Auf dieser Basis gelang es 1966, den Rahmen für ein langfristiges Arbeitsprogramm abzustecken und in langwierigen Verhandlungen die Voraussetzungen für den Aufbau der Dokumentationsstelle zu schaffen, deren Gründung allerdings erst 1967 erfolgte. In diesem Jahr konnten die Mitarbeiter des Instituts die für ihre Arbeit erforderlichen Afrika-Reisen durchführen und zugleich das Institut auf wichtigen internationalen Veranstaltungen wie der Conference on African Bibliography in Nairobi (4. bis 8. 12.) und dem Zweiten Internationalen Afrikanistenkongreß in Dakar (10. bis 20. 12.) vertreten. Die Ergebnisse der bisher geleisteten Arbeit konnten in den sechs seit 1965 erschienenen Monographien der Reihe Hamburger Beiträge zur Afrika-Kunde, den sechs Heften des Afrika Spectrums sowie in dem 1967 veröffentlichten ersten Teil des Afrika-Handbuchs vorgelegt werden.

Auf dem Gebiet der Rechtswissenschaft stehen die Untersuchungen über Fragen des Niederlassungsrechts im Vordergrund. Die 1965 herausgegebene erste Studie behandelt die Grundzüge des Niederlassungsrechts in den afrikanischen Staaten französischer Prägung 4 . Nach einer gerafften Zusammenfassung der deutschen Bestimmungen zur Förderung von Kapitalanlagen im Ausland werden die Rechtsvorschriften, die ein ausländischer Privatinvestor in den frankophonen Staaten südlich der Sahara und in den drei nordafrikanischen Staaten Algerien, Marokko und Tunesien zu beachten hat, erörtert. Die Fragen des Grunderwerbs und des Enteignungsschutzes, des gewerblichen Rechtsschutzes, des Kapital- und Gewinntransfers und des Gesellschaftsrechts werden in ihren wesentlichen Grundzügen dargestellt. Den Vergünstigungen, die auf steuerlichem und zollrechtlichem Gebiet privaten Investoren teils nach Vorschriften des allgemeinen Rechts, teils in besonderen Investitionsgesetzen eingeräumt werden, ist ein besonderer Hauptteil der Arbeit gewidmet. Die entsprechenden Vorschriften für den größten Teil der englischsprachigen Staaten Afrikas werden im zweiten Teil dieser Studie untersucht, deren Veröffentlichung Mitte 1968 vorgesehen ist. Weitere Studien sind über das Niederlassungsrecht in den übrigen Staaten, darunter insbesondere Äthiopien, Liberia, Libyen und Somalia; Burundi, Kongo-Kinshasa und Ruanda (ehemals belgische Gebiete); Botswana, Lesotho, Südafrika, Südwestafrika und Swasiland (vom „Roman-Dutch law" beeinflußter Rechtskreis) sowie Angola und Mozambique (portugiesische Gebiete) geplant. Von besonderer Bedeutung ist daneben die laufende Dokumentation der afrikanischen Gesetzgebung in den Heften des Afrika Spectrums. Um sie noch benutzbarer zu gestalten, wird ab 1968 ein systematischer Jahres-Index zusammengestellt, dessen erste Ausgabe für die Jahrgänge 1966 und 1967 als Beilage zu Heft 1/1968 erscheinen wird. Im Rahmen des Afrika Spectrums ${ }^{5}$ erschienen die folgenden Aufsätze: Wolfgang Heidelberg, "Gedanken zum Recht" (in Botswana und Lesotho) (1/1966) sowie Dieter Schröder, Forschungsstelle für Völkerrecht, Universität Hamburg, „Kamerun - Modell eines afrikanischen Bundesstaates?" (1/1967).

Im Bereich der Wirtschafts- und Sozialwissenschaften wurden verschiedene Themenkreise angeschnitten. Einmal sollen Rentabilitätsstudien zur Aussonderung wirtschaftlich toter und potentiell nutzbarer Räume in Afrika angefertigt werden. Andererseits ist die Darstellung der See- und Küstenschiffahrt afrikanischer Staaten vorgesehen. Als Modell dient die 1967 vorgelegte Studie über die Wirtschaftsräume Angolas. Aufgabe dieser Arbeit war es, ausgehend von der Transportbelastung wirtschaftliche Vermarktungsgrenzen einzelner Produkte im Hinterland der Häfen

4 Näheres über Verfasser und genauen Titel vgl. S. 252.
5 Genaue Titel vgl. S. 252 . 
und der Bahneinzugsbereiche in Angola zu erfassen. Dabei ergab sich, daß es für jedes Massengut einen Grenzbereich gibt, aus dem das betreffende Produkt noch konkurrenzfähig auf den Weltmarkt gelangen kann. Weitere Studien dieser Art, deren Ergebnisse in einem ausführlichen Karten-Anhang veranschaulicht werden, werden zur Zeit von Gerhard Oberbeck, Geographisches Institut der Universität Hamburg, über Kamerun und von Günter Borchert, Geographisches Institut der Universität Hamburg, über die Elfenbeinküste durchgeführt.

Als Modell für die Arbeiten über die See- und Küstenschiffahrt dient die Studie Angola und seine Seehäfen. Hier wird der Versuch unternommen, die auslösenden Momente, die den Aufstieg der Häfen Luanda, Lobito und Moçâmedes zu wichtigen afrikanischen Regionalhäfen bewirkten, darzustellen und darüber hinaus mögliche Tendenzen aufzuzeigen, welche die weitere Entwicklung der Häfen nachhaltig beeinflussen können. Ein besonderes Anliegen ist dabei, die vielfältigen geographischen Sachverhalte transparent $\mathrm{zu}$ machen, die bei einer ausschließlich verkehrswirtschaftlich ausgerichteten Hafenuntersuchung nicht die Gewichtung erhalten, die ihnen gerade in einem tropisch-afrikanischen Entwicklungsland zukommen. Diese werden besonders bei der Analyse der produktionswirtschaftlichen Voraussetzungen für die Entwicklungstendenzen des Seehandels der einzelnen Häfen deutlich, die sich jeweils an die Untersuchung des Seehafenverkehrs und der Zusammenhänge zwischen Verkehrs- und baulicher Entwicklung der Häfen anschließt. Neben den drei angolanischen Haupthäfen werden auch eine Reihe von Sekundärhäfen einer Analyse unterzogen sowie auf Fragen der Küstenschiffahrt eingegangen, die bei der verkehrswirtschaftlichen Verklammerung der Hinterlandsbereiche von Luanda, Lobito und Moçâmedes eine nicht unerhebliche Rolle spielt. Auch diese Arbeit ist mit vorzüglichem Kartenmaterial ausgestattet.

Für produktionswirtschaftliche Fragen wird an Hand einer Studie über den Kakao ein Modell für die Untersuchung der wichtigsten afrikanischen Rohstoffe erarbeitet. Besondere Berücksichtigung erfährt dabei die Darstellung der Bedeutung und Stellung einzelner tropischer Exportprodukte in den Volkswirtschaften der afrikanischen Räume. In diesem Zusammenhang soll auch zu den Problemen der Diversifikation tropischer Monokulturen Stellung genommen werden. Im Rahmen des Afrika Spectrums wurden u. a. folgende Aufsätze vorgelegt: Klaus Dahms, Firma H. O. Lütkens in Hamburg, "Die Forst- und Holzwirtschaft in Kamerun“ (1/1967); Hansgünter Schönwälder, „Zur Rentabilität des Reisanbaus in Westafrika“ (1/1968); Detlev Karsten, Universität Addis Abeba, „Die Ensete (falsche Banane) - ein Beispiel zur Ernährungswirtschaft" (1/1968).

Die für die zwischenstaatlichen Beziehungen wie für die Entwicklungspolitik in Afrika bedeutsamen Fragen der zwischenstaatlichen Zusammenarbeit und der Integration wurden in den Themenkreis einbezogen. Ziel der Untersuchungen ist die systematische Erfassung und Darstellung der vielfältigen Ansätze auf diesem Gebiet. Schwerpunkt sind hierbei die Bereiche der wirtschaftlichen und technischen Zusammenarbeit. Zunächst sind Studien über die zentralafrikanische Subregion und über die Ansätze zur Zusammenarbeit im französischgeprägten Westafrika vorgesehen. Im Afrika Spectrum erschienen zu diesem Thema die Aufsätze von Harald Voss, „Probleme der wirtschaftlichen Zusammenarbeit im südlichen Afrika“ (1/1966), und Ulrich Stipke, Vereinsbank Hamburg, „Probleme monetärer Integration in Afrika" (2/1967). Weitere Beiträge befinden sich für das Integrationsfragen gewidmete Heft 2/1968 in Vorbereitung.

Wirtschaftliche Themen waren Gegenstand zahlreicher Aufsätze im Afrika Spectrum: 1/1966: Martin Krämer, „Bemerkungen zur wirtschaftlichen Entwicklung 
und zum Außenhandel Botswanas“; Hansgünter Schönwälder, „Die Viehwirtschaft Botswanas“; Georg Heuß „Voraussetzungen und Möglichkeiten der wirtschaftlichen Entwicklung Lesothos“. 1/1967: Philipp Risch, „Makroökonomische Daten der Entwicklungsplanung “ (Kameruns); 2/1967: Peter Thomas Bauer, London School of Economics, „UNCTAD and Africa“; 3/1967: Oskar Alber, Hamburg, „Erdöl und Erdgas der Sahara“; 1/1968: Ida Bernier und R. Bousser, Institut pour la technologie et l'industrialisation des produits agricoles tropicaux, Abidjan, „Intensive Industrialisierung des Landwirtschaftserzeugnisses und Herstellung ,neuartiger Fertigprodukte"; Rolf Steinberg, Bundesministerium für Ernährung, Landwirtschaft und Forsten, Bonn, „Die Bedeutung der Fischerei für die Ernährungswirtschaft Westafrikas"; Henriette Oboussier und Reinmar Grimm, Zoologisches Staatsinstitut, Universität Hamburg, „Probleme des Naturschutzes und der Wildhege in Afrika; Hans Dietrich Cremer, Institut für Ernährungswissenschaft, Universität Gießen, „Grundfragen der Ernährungswirtschaft in Afrika“.

Im Rahmen seines Forschungsprogramms ist das DIAF darum bemüht, die Entwicklung der Massenmedien in Afrika im einzelnen zu untersuchen. 1966 wurde zunächst die Studie über die arabische Presse in den Maghreb-Staaten veröffentlicht. Den besonderen Anforderungen des Themas entsprechend, war ein Angehöriger des arabischen Sprachraums mit der Durchführung betraut worden. Ausführlich setzt sich der Autor mit dem Problem der Pressefreiheit auseinander, die in allen vier Maghreb-Staaten als Grundsatz in der Pressegesetzgebung verankert ist, in der Praxis aber gewissen Einschränkungen unterliegt. Die arabischsprachige und die fremdsprachige Presse in einheimischer und in ausländischer Hand sowie das Anzeigen- und das Nachrichtenwesen werden im einzelnen analysiert. Auch die anderen Massenmedien werden in die Untersuchung einbezogen. Sie kommt zu dem Ergebnis, daß die fremdsprachige Presse in den Maghreb-Staaten noch immer eine Vorrangstellung besitzt, während der stärkste Einfluß nach wie vor vom Rundfunk ausgeübt wird.

Aufgabe der zweiten Studie dieses Themenkreises war die Untersuchung der Presse in Westafrika. Hier wird zunächst in einem systematischen Teil die Lage der Presse in diesem Raum dargestellt und in einem hervorragenden Tabellenteil übersichtlich zusammengefaßt. Die Eigentumsverhältnisse, die Herstellung sowie die Verflechtungen in der Verlagswirtschaft, Vertriebsprobleme, die Zeitungssprachen und das Anzeigengeschäft werden besonders abgehandelt. Eigene Abschnitte befassen sich mit dem Nachrichtenwesen und der Journalistenausbildung. Weitere Hauptteile der Arbeit bilden die nach Staaten bzw. Staatengruppen gegliederte Geschichte des Pressewcsens in Westafrika und die Untersuchung der Beziehungen zwischen Presse und Staatsmacht. Als wichtigstes Ergebnis werden die starken Unterschiede in der Entwicklung des Pressewesens in den einzelnen Staaten und das starke Gefälle zwischen den britisch und den französisch geprägten Gebieten auf diesem Sektor herausgearbeitet.

Außerdem wurden die Massenmedien in verschiedenen Aufsätzen des Afrika Spectrums behandelt: Hans Ulrich Behn, Bundespresseamt in Bonn, „Das Zeitungswesen in Ostafrika“; Harald Voss, „Rundfunk und Fernsehen in Ostafrika“ (2/1966). Hans Ulrich Behn, „Das Pressegesetz in Kamerun“; Harald Voss, „Der Kameruner Rundfunk“ (1/1967). Im Rahmen des Forschungsprogrammes sind weitere Studien über die Presse in Zentral- und Südafrika sowie über Rundfunk und Fernsehen in Afrika vorgesehen.

Einen wesentlichen Anteil an der Forschungsarbeit des Instituts haben die geographischen Disziplinen. Außer den bereits in anderem Zusammenhang erwähn- 
ten Studien ist hier die von Ernest Troger, Geographisches Institut der Universität Wien, übernommene bevölkerungsgeographische Studie über den Einfluß der Arbeiterwanderungsbewegung auf Kultur und Wirtschaft des malawischen Dorfes zu nennen. Die Aufgabe dieser Untersuchung besteht darin, die Wechselwirkungen zwischen industrialisierten und rein agrarischen Räumen im zentralen und südlichen Afrika an Hand dieses Teilproblems herauszuarbeiten. Außerdem soll der Einfluß der Wanderbewegung auf Strukturveränderungen im ländlichen Lebensraum eines zentralafrikanischen Landes ermittelt werden. Im Afrika Spectrum wurden die folgenden Aufsätze veröffentlicht: Robert Martens, Geograpisches Institut der Universität Hamburg, „Naturgeographische Grundlagen Botswanas“ und „Natur und Landschaft Lesothos" (1/1966); Eberhard Klitzsch, Institut für Geologie und Palätologie an der Technischen Universität Berlin, „Über den Grundwasserhaushalt der Sahara "; Horst Hagedorn, Geographisches Institut Berlin Dahlem, „Siedlungsgeographie des Sahara-Raumes“ (3/1967). Als besonderer Beitrag sind ferner die Karten, die für verschiedene Publikationen des DIAF in enger Zusammenarbeit mit dem Geographischen Institut Hamburg angefertigt wurden, hervorzuheben.

Historische und prähistorische Themen wurden bisher nur im Rahmen des Afrika Spectrums behandelt: Günther Jantzen, „Zur Geschichte von Lesotho und Botswana“; Peter Colberg, „Verfassungsentwicklung und Politik“ (in Botswana und Lesotho) (1/1966); Helmut Ziegert, Seminar für Vor- und Frühgeschichte der Universität Hamburg, „Zur Pleistozän-Gliederung in Nordafrika“ (3/1967).

Auf dem Gebiet der Sprach- und Kulturwissenschaft ist aus besonderem Anlaß eine Sammelarbeit, die Beiträge von 28 namhaften Afrikanisten aus dem In- und Ausland vereinigt, unter dem Titel „Neue Afrikanische Studien“ erschienen. Sie ist als Festschrift dem 1967 verstorbenen Nestor der deutschen Afrika-Forschung, Professor Dr. August Klingenheben, zum 80. Geburtstag gewidmet. Die Aufsätze gehen auf die verschiedenen, Professor Klingenheben durch seine Forschungen vertrauten Räume in Afrika ein, davon insbesondere die Gebiete nördlich der afrikanischen Regenwaldzone. Daneben finden sich auch Beiträge zur Vai-Forschung, über die sprachlichen Beziehungen zwischen Ägypten und Afrika sowie zur Bantuistik und ihren Randgebieten, zum Nama-Hottentottischen und zum Studium der Khoisan-Sprachen. Damit ist in diesem Werk ein eindrucksvoller Querschnitt durch die Afrikanistik gegeben worden.

Wichtige Anregungen für seine Arbeit empfing das Institut aus den Colloquien, in denen insbesondere die Möglichkeiten interdisziplinärer Zusammenarbeit erörtert wurden. Bisher standen die folgenden Themen zur Diskussion: Zur Afrika-Wissenschaft und Afrika-Forschung in den USA - Grundlagen und Motive eines modernen Afrika-Bildes; Die Wirtschaftsräume Angolas und ihre Probleme; Aspekte des modernen Äthiopien - eine kulturwissenschaftliche Betrachtung; die Häfen Angolas im Spiegelbild der wirtschaftlichen Entwicklung; zur PleistozänGliederung in der zentralen Sahara (Ostfezzan und Nord-Tibesti); die wirtschaftliche Gliederung Kameruns - ihre Bedeutung für Produktion, Transport und Investierung; die hauptsächlichen Kulturkreise in Südafrika und das Problem der Akkulturation; Fragen und Erfordernisse einer afrika-wissenschaftlichen Dokumentation; Welthandelskonferenz und Afrika; die Völkerkunde und ihre Stellung in der interdisziplinären Afrika-Forschung; die Arbeiterwanderbewegung in Malawi. $\mathrm{Zu}$ den besonderen Aufgaben des DIAF gehört die Pflege enger Kontakte mit der Afrika-Forschung im In- und Ausland. Das Institut ist Mitglied der Ende 1967 gegründeten Arbeitsgemeinschaft der deutschen Afrika-Forschungs- und Dokumentationsstellen, von der es zusammen mit der Friedrich-Ebert-Stiftung in Bonn und 
der Afrika-Forschungsstelle des IFO-Instituts in München in den Vorstand berufen wurde. Durch die Dokumentationsarbeit steht es in engem Kontakt zur CIDESA in Brüssel und zahlreichen anderen Stellen. Seit 1967 ist das DIAF Mitglied des International African Institute in London. Außerdem konnten die Beziehungen zu Forschungsinstitutionen in Afrika stark ausgebaut werden, auf die seinem Auftrag entsprechend von Anfang an besonderer Wert gelegt wurde.

\section{VERÖFFENTLICHUNGEN}

\section{HAMBURGER BEITRÄGE ZUR AFRIKA-KUNDE}

Band 1 Wolfgang Heidelberg, Grundzüge des Niederlassungsrechts in den afrikanischen Staaten. Teil 1 (ehem. französische Gebiete). Hamburg 1965, 228 S., 18,- DM

Band 3 Ahmed Muddathir, Die arabische Presse in den Maghreb-Staaten. Hamburg 1966, 148 S., 18,- DM

Band 5 Johannes Lukas (Hrsg.), Neue afrikanische Studien. Hamburg 1966, 319 S., 48,- DM

Band 6 Günter Borchert, Die Wirtschaftsräume Angolas - Transportprobleme und Rentabilitätsgrenzen der Produktion. Hamburg 1967, 143 S., 4 Karten, 22,- DM

Band 7 Gerhard Sendler, Angola und seine Seehäfen - weltwirtschaftlich relevante Seehäfen, Küstenschiffahrtsplätze und Küstenschiffahrt. Hamburg 1967, 148 S., 6 Karten, 22,- DM

Band 8 Hans Ulrich Behn, Die Presse in Westafrika. Hamburg 1968, 264 S., $22,-\mathrm{DM}$

\section{AFRIKA SPECTRUM}

Jahresabonnement (3 Hefte) DM 24,-, Einzelheft DM 8,50

abonnement (3 Hefte) DM 24,-, Einzelheft DM 8,50.

Heft 1/1966 Neue Staaten Afrikas: Botswana und Lesotho

Heft 2/1966 Presse und Funk in Ostafrika

Heft 1/1967 Politische und wirtschaftliche Fragen Kameruns

Heft 2/1967 Welthandelskonferenz, Währungspolitik und Afrika

Heft 3/1967 Die Sahara

Heft 1/1968 Fragen der Ernährungswirtschaft in Afrika

Auslieferung über Afrika-Verlag, 8068 Pfaffenhofen, Postfach 86

H. V. 